\title{
A Threat Assessment Model under Uncertain Environment
}

\author{
Yong Deng \\ School of Electronics and Information, Northwestern Polytechnical University, Xian, Shaanxi 710072, China \\ Correspondence should be addressed to Yong Deng; ydeng@nwpu.edu.cn
}

Received 30 April 2015; Revised 13 July 2015; Accepted 29 July 2015

Academic Editor: Kyandoghere Kyamakya

Copyright (C) 2015 Yong Deng. This is an open access article distributed under the Creative Commons Attribution License, which permits unrestricted use, distribution, and reproduction in any medium, provided the original work is properly cited.

\begin{abstract}
Threat evaluation is extremely important to decision makers in many situations, such as military application and physical protection systems. In this paper, a new threat assessment model based on interval number to deal with the intrinsic uncertainty and imprecision in combat environment is proposed. Both objective and subjective factors are taken into consideration in the proposed model. For the objective factors, the genetic algorithm (GA) is used to search out an optimal interval number representing all the attribute values of each object. In addition, for the subjective factors, the interval Analytic Hierarchy Process (AHP) is adopted to determine each object's threat weight according to the experience of commanders/experts. Then a discounting method is proposed to integrate the objective and subjective factors. At last, the ideal of Technique for Order Preference by Similarity to Ideal Solution (TOPSIS) is applied to obtain the threat ranking of all the objects. A real application is used to illustrate the effectiveness of the proposed model.
\end{abstract}

\section{Introduction}

In the real world, it is always complex and difficult to make a decision and to deal with threat assessment in most situations, because the problems we are faced with are always intrinsic imprecision and uncertainty which is typically represented by the threat assessment for multiobject and multiattribute with uncertain information. Hence, it is meaningful to develop a reasonable model to solve this problem. The threat assessment of multiobject and multiattribute has been researched by many researchers. Generally speaking, threat assessment can be seen as a decision-making problem under uncertain environment. We first introduce some related works about decision making and threat assessment.

1.1. Decision Making. Decision making has been the popular topic of research in many fields since the concept was proposed. Saaty $[1,2]$ introduces the AHP which is a multicriteria decision-making approach in which factors are arranged in a hierarchic structure. Hall and Davis [3] introduce a valuebased decision-making model which suggests that multiple perspectives may be achieved by considering a foundation of individual values. Zhang et al. [4] present a method for solving the stochastic MCDM problem and propose a new concept of stochastic dominance degree (SDD). Chiadamrong
[5] introduces the concept of fuzzy set theory to overcoming the precision-based evaluation for manufacturing strategies selection. Hon et al. [6] make use of a procedure to solve the multiple attributes and multiple hierarchical system under fuzzy environment. This procedure is based on gradient eigenvector method or rating the fuzzy criteria weighting and the max-min paired elimination method for aggregation. Forgionne and Kohli [7] present a methodology that objectively rates decision technology system journals across multiple and relevant dimensions. Through this process, the AHPbased rating model and the DSS-delivery mechanism are the major contribution. Fan et al. [8] propose a method based on pairwise comparisons of alternatives with random evaluations to solve stochastic MCDM problem. By computing the probabilities of all possible results of pairwise comparisons and using the identification rule, superior, indifferent, and inferior probabilities on pairwise comparisons of alternatives are estimated. Al-Najjar and Alsyouf [9] make use of the advantage of both fuzzy logic and multiple criteria decision making to introduce an evaluation methodology. Wang [10] proposes a new multiple criteria decision-making method with incomplete certain information based on ternary AHP; in this process, the ternary comparison matrix of the alternatives is constructed to normalize priority vector of the alternatives. Similar researches are conducted by Chan et al. 
[11]. Due to the efficient modelling and handling of uncertain information, fuzzy set theory [12-15] and evidence theory are also widely used in decision making [16-23]. Some typical evidence theories with AHP are published in [24, 25].

1.2. Threat Assessment. A threat is defined as any act, entity, event, or phenomenon with the potential to harm a person or thing. In other words, a threat is a source of potential harm. Sometimes the word hazard or risk is used as a synonym for threat. The term "threat assessment" can be broadly interpreted as evaluation of impending danger or harm by a person, group, circumstance, or set of conditions. It is a pattern of activities involving detection and analysis of threat stimuli and the situations in which the threat is encountered. Threat or risk assessment serving as the foundation of regulatory decision making whether to take actions to reduce the loss has been researched by many researchers. Pedrycz et al. [26] introduce a scheme of risk assessment on a basis of classification results produced for some experimental data capturing the history of previous threat cases. Through this procedure, information granulation (fuzzy clustering) was used to reveal the structural relationships in the experimental data. van de Walle and Rutkowski [27] designed and developed FURIA (fuzzy relational incident analysis), a prototype fuzzy decision support system allowing individual group members to compare their individual assessment of a decision alternative to the assessment of the other group members. Looney and Liang [28] propose a multiphase approach to middle and high level data fusion with an application to situation and threat assessment, threat assessment with fuzzy belief network. Khadam and Kaluarachachi [29] propose a decision analysis framework which integrates probabilistic health risk assessment into comprehensive, yet simple, cost-based multicriteria decision analysis framework. Assmuth and Hildén [30] present an analysis of frameworks for information on integrated risk assessment and management, which focuses on issues related to environmental and health risks of chemicals and their regulation at the EU level. Chen and $\mathrm{Ma}$ [31] propose a method combining the cost of reducing uncertainty with the selection of risk assessment models for remediation decision of site contamination; in this study, they explore how to choose among equally plausible models for risk-related decisions, which is closely related to model uncertainty. Jeong et al. [32] introduce a risk assessment method which is developed by using risk matrix and fuzzy inference logic, on the basis of the radiological and nonradiological hazard for a decommissioning safety of a nuclear facility. Bonano et al. [33] developed and demonstrated a single framework integrating risk assessment and decision analysis methods for evaluating, ranking, and selecting preferred remediation alternatives at a contaminated site; in the decision analysis framework, the Analytic Hierarchy Process was selected as a technique to obtain stakeholder working group input. van Duijne et al. [34] outline the preconditions for risk assessment method and aim to clarify the difficulties and pitfalls in the subsequent stages of risk assessment. Kalantarnia et al. [35] demonstrate the use of Bayesian theory in quantitative risk assessment (QRA) and its application as a useful tool in dynamic risk assessment to prevent accidents, which overcomes the major disadvantages of QRA: its inability to update risk during the life of a process. In the past few years, the field of group decision and threat evaluation has been researched hotly, like in Deng et al. [36].

Evaluation for aerial objects is an important part in the aerial defensive operation, which can be described as the assessment of multiobject and multiattribute under uncertain environment. Due to the limitation of detection means or secrecy, the effective information we can obtain from the threat objects is little. Generally, the interval number can be used to represent the uncertain or imprecise information. In this paper, a new model is proposed to rank the threat objects based on interval numbers, which takes the subjective and objective factors into consideration. Two examples are used to illustrate the effectiveness of the proposed threat evaluation model.

This paper is organized as follows. Section 2 mainly introduces several concepts of interval numbers, the classical genetic algorithm, interval AHP, and the TOPSIS. Section 3 introduces the model of threat assessment in detail. Section 4 introduces a simple numerical example and a real application to illustrate the effectiveness of the proposed assessment model. A conclusion is made in Section 5.

\section{Preliminaries}

As mentioned above, there are many math tools to handle uncertain information, such as fuzzy sets theory [37-40] and evidence theory [41]. In addition, interval number is also a simple and efficient method. In this section, the basic concepts of interval number are briefly introduced.

2.1. Interval Number. The interval approach is originally developed by Moore $[42,43]$ in order to keep track of the lower and upper bounds to the exact result when carrying out numerical calculation on digital computers with a finite number of significant digits. Recently, it has been suggested to use interval numbers to represent the uncertainty or probability resulting from instinct of the incomplete knowledge or cognition [44-46]. In this paper, interval numbers are used to represent the uncertain value of threat attributes.

Definition 1. Interval number is defined as an ordered pair $\tilde{a}=\left[a^{-}, a^{+}\right]=\left\{x \mid a^{-} \leq x \leq a^{+}, a^{-}, a^{+} \in R\right\}$; particularly, if $a^{-}=a^{+}$, then $\tilde{a}$ is obviously equal to a real number, and the interval numbers we discussed in this paper are all positive interval numbers which can be described as $\tilde{a}=\left[a^{-}, a^{+}\right]=$ $\left\{x \mid a^{-} \leq x \leq a^{+}, a^{-}, a^{+} \in R^{*}\right\}$, where $R^{*}$ is positive real number set.

Definition 2. Let $a=\left[a^{-}, a^{+}\right], b=\left[b^{-}, b^{+}\right]$be any two interval numbers; then some of their arithmetic operations are defined as follows:
(1) $a=b$ if and only if $a^{-}=b^{-}$and $a^{+}=b^{+}$;
(2) $a+b=\left[a^{-}+b^{-}, a^{+}+b^{+}\right]$. 
The problem of a multiobjective threat assessment can be described with the mathematical model. The object set can be represented with $R=\left\{R_{1}, \ldots, R_{m}\right\}$ and the attribute set is $F=\left\{F_{1}, \ldots, F_{n}\right\}$. Decision matrix $R=\left(r_{i j}\right)_{m \times n}$ is composed of attribute values (interval number $r_{i j}=\left[r_{i j}^{-}, r_{i j}^{+}\right], i=1, \ldots, m$; $j=1, \ldots, n)$. The threat goals $R_{i}$ are measured by the attribute $F_{j}$. And the attribute $F_{j}$ is supplied by specific researches according to the different situation.

Definition 3. The attribute of each object is composed of two types, namely, "cost type" and "benefit type." Let $J_{1}, J_{2}\left(J_{1} \cup\right.$ $J_{2}=n$ ) represent their corresponding subscript set, and the "cost type" is a kind of attribute which becomes better with its value increasing while "benefit type" is a kind of attribute which becomes better with its value decreasing. To eliminate the influence of decision results in different physical dimension, $R$ is normalized to $(X)_{m \times n}$ with the procedure defined as follows:

$$
\begin{aligned}
& x_{i j}^{-}=\frac{r_{i j}^{-}}{\sqrt{\sum_{i=1}^{n}\left(r_{i j}^{+}\right)^{2}}}, \\
& x_{i j}^{+}=\frac{r_{i j}^{+}}{\sqrt{\sum_{i=1}^{n}\left(r_{i j}^{-}\right)^{2}}}, \\
& x_{i j}^{-}=\frac{1 / r_{i j}^{+}}{\sqrt{\sum_{i=1}^{n}\left(1 / r_{i j}^{-}\right)^{2}}}, \\
& x_{i j}^{+}=\frac{1 / r_{i j}^{-}}{\sqrt{\sum_{i=1}^{n}\left(1 / r_{i j}^{+}\right)^{2}}},
\end{aligned}
$$$$
i \in N, j \in J_{1} \text {, }
$$

$$
i \in N, j \in J_{2} \text {. }
$$

Definition 4. Let $F(R)$ be the set of interval numbers in $R$, and let the distance between two interval numbers $A\left(a_{1}, a_{2}\right)$ and $B\left(b_{1}, b_{2}\right)$ be defined by Tran and Duckstein [47] as

$$
\begin{aligned}
& D^{2}(A, B)=\int_{-1 / 2}^{1 / 2}\left\{\left[\left(\frac{a_{1}+a_{2}}{2}\right)+x\left(a_{2}-a_{1}\right)\right]\right. \\
& \left.-\left[\left(\frac{b_{1}+b_{2}}{2}\right)+x\left(b_{2}-b_{1}\right)\right]\right\}^{2} d x, \\
& D^{2}(A, B)=\left[\left(\frac{a_{1}+a_{2}}{2}\right)-\left(\frac{b_{1}+b_{2}}{2}\right)\right]^{2}+\frac{1}{12}\left[\left(a_{2}\right.\right. \\
& \left.\left.-a_{1}\right)+\left(b_{2}-b_{1}\right)\right]^{2} .
\end{aligned}
$$

It can be proved that $D(A, B)=\sqrt{D^{2}(A, B)}$ is a distance on $F(R)$. First, $D(A, B) \geq 0$. Symmetry is transparent. If $D(A, B)=0$, then $A=B$. The triangle inequality follows from the fact that the function to be integrated in (2) is the square of Euclidean distance.

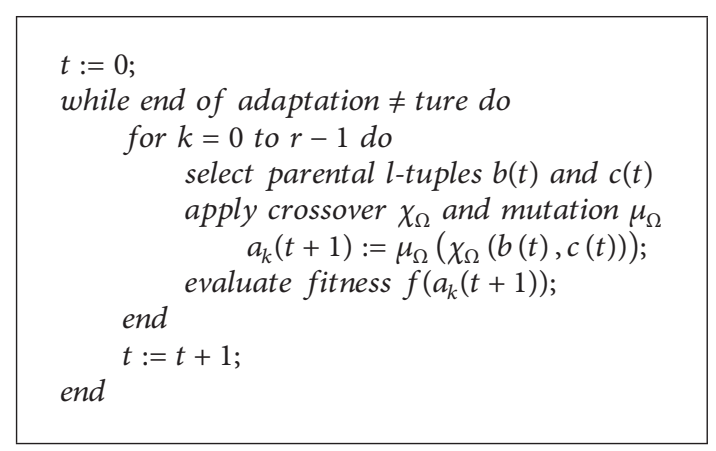

Algorithm 1: \% procedure: simple genetic algorithm.

Although only the lower and upper bound values of the two interval numbers appear in (3), which is derived from (2) for operational purpose, the integral in (2) shows that this distance takes into account every point in both intervals when computing the distance between those two interval numbers. It is different from most existing distance measures for interval numbers which often use only the lower and upper bound values [48-50]. Paper [51] proposes a distance measure for intervals which also considers every point of both intervals. Its general form, however, is too complicated and the authors later restricted the measure to a particular case with a finite number of considered values for operational purpose.

2.2. Genetic Algorithms. The simple genetic algorithm (Algorithm 1) over populations defined as multisets $P(t)=\left(a_{0}(t), a_{1}(t), \ldots, a_{r-1}(t)\right)$ consists of $r$ individual binary l-tuples $a_{k}(t)=\left(a_{k, 0}(t), a_{k, 1}(t), \ldots, a_{k, l-1}(t)\right) \in \Omega$ with fitness values $f\left(a_{k}(t)\right)$. For the creation of offspring individual in each generation $t$ random genetic operators like crossover $\chi_{\Omega}$ and mutation $\mu_{\Omega}$ are applied to parental individuals which are selected according to their fitness values as follows. The population $P(0)$ is initialised appropriately, for example, by randomly choosing individuals in $\Omega$. GA is also used to cope with the issue of decision making by lots of researchers like Chan et al. [52].

(1) Selection. For fitness-proportional selection each individual $a_{k}(t)$ in the population $P(t)$ is selected with a probability $p_{k}(t)$ which is directly proportional to its fitness $f\left(a_{k}(t)\right) \geq 0$. The individual selection probability is then given by

$$
p_{k}(t)=\frac{f\left(a_{k}(t)\right)}{\sum_{j=0}^{r-1} f\left(a_{j}(t)\right)} .
$$

(2) Crossover. The crossover operator $\chi_{\Omega}: \Omega \times \Omega \rightarrow \Omega$ takes two selected individual $l$-tuples $a=\left(a_{0}, a_{1}, \ldots, a_{l-1}\right)$ and $b=$ $\left(b_{0}, b_{1}, \ldots, b_{l-1}\right)$ and randomly generates an offspring $l$-tuple $c=\left(c_{0}, c_{1}, \ldots, c_{l-1}\right)$ according to $c=\chi_{\Omega}(a, b)$.

For $l$-pointcrossover a position $1 \leq \lambda \leq l-1$ is chosen with uniform probability and the two $l$-tuples $\left(a_{0}, \ldots, a_{\lambda-1}\right.$, $\left.b_{\lambda}, \ldots, b_{l-1}\right)$ and $\left(b_{0}, \ldots, b_{\lambda-1}, a_{\lambda}, \ldots, a_{l-1}\right)$ are generated, one of which is randomly chosen as offspring $c$. The 1-point 
crossover operator is applied with crossover probability $\chi$. In case of uniform crossover each bit of the offspring $c$ is chosen with probability $\chi$ from the parental $l$-tuple $a$ and with probability $1-\chi$ from the parental $l$-tuple $b$ or vice versa.

(3) Mutation. In the simple genetic algorithm with binary $l$ tuples the bitwise mutation operator $\chi_{\Omega}: \Omega \rightarrow \Omega$ is defined by randomly flipping each bit of the individual $l$-tuple $a=$ $\left(a_{0}, a_{1}, \ldots, a_{l-1}\right)$ with small mutation probability $\mu$. A typical value is $\mu \leftrightarrow 1 / l$.

2.3. Interval $A H P$. Assume that a decision maker provides interval judgements instead of precise judgements for a pairwise comparison matrix. For example, it could be judged that $i$ th criterion is between $a_{i j}^{L}$ and $a_{i j}^{U}$ times as important as $j$ th criterion with $a_{i j}^{L}$ and $a_{i j}^{U}$ being nonnegative real numbers and $a_{i j}^{L} \leq a_{i j}^{U}$. Then, an interval comparison matrix can be expressed as

$$
\widetilde{A}=\left[\begin{array}{cccc}
{\left[a_{11}^{L}, a_{11}^{U}\right]} & {\left[a_{12}^{L}, a_{12}^{U}\right]} & \cdots & {\left[a_{1 n}^{L}, a_{1 n}^{U}\right]} \\
{\left[a_{21}^{L}, a_{21}^{U}\right]} & {\left[a_{22}^{L}, a_{22}^{U}\right]} & \cdots & {\left[a_{2 n}^{L}, a_{2 n}^{U}\right]} \\
\vdots & \vdots & \vdots & \vdots \\
{\left[a_{n 1}^{L}, a_{n 1}^{U}\right]} & {\left[a_{n 2}^{L}, a_{n 2}^{U}\right]} & \cdots & {\left[a_{n n}^{L}, a_{n n}^{U}\right]}
\end{array}\right],
$$

where $a_{i j}^{L}=1 / a_{j i}^{U}$ and $a_{i j}^{U}=1 / a_{j i}^{L}$ for all $i, j=1 \cdots n$. The above interval comparison matrix can be split into two crisp nonnegative matrices:

$$
\begin{aligned}
A^{L} & =\left[\begin{array}{cccc}
a_{11}^{L} & a_{12}^{L} & \cdots & a_{1 n}^{L} \\
a_{21}^{L} & a_{22}^{L} & \cdots & a_{2 n}^{L} \\
\vdots & \vdots & \vdots & \vdots \\
a_{n 1}^{L} & a_{n 2}^{L} & \cdots & a_{n n}^{L}
\end{array}\right], \\
A^{U} & =\left[\begin{array}{cccc}
a_{11}^{U} & a_{12}^{U} & \cdots & a_{1 n}^{U} \\
a_{21}^{U} & a_{22}^{U} & \cdots & a_{2 n}^{U} \\
\vdots & \vdots & \vdots & \vdots \\
a_{n 1}^{U} & a_{n 2}^{U} & \cdots & a_{n n}^{U}
\end{array}\right],
\end{aligned}
$$

where $\widetilde{A}=\left[A^{L}, A^{U}\right]$.

Let $\lambda_{\max }=\left[\lambda_{\max L}, \lambda_{\max U}\right]$ be the principal eigenvalue of $\widetilde{A}$, which is an interval number. The principal right eigenvector corresponding to $\lambda_{\max }$ is denoted by $W=\left(\left[\omega_{1}^{L}, \omega_{1}^{U}\right]\right.$, $\left.\ldots,\left[\omega_{n}^{L}, \omega_{n}^{U}\right]\right)^{T}=\left[W_{L}, W_{U}\right]$, where $W_{L}=\left(\omega_{1}^{L}, \ldots, \omega_{n}^{L}\right)^{T}$ and $W_{U}=\left(\omega_{1}^{U}, \ldots, \omega_{n}^{U}\right)^{T}$. Therefore, the eigenvalue problem can be written as

$$
A W=\lambda_{\max } W
$$

namely,

$$
\left[A_{L}, A_{U}\right]\left[W_{L}, W_{U}\right]=\left[\lambda_{\max L}, \lambda_{\max U}\right]\left[W_{L}, W_{U}\right] .
$$

According to interval arithmetic, (8) can be expressed as

$$
\left[A_{L} W_{L}, A_{U} W_{U}\right]=\left[\lambda_{\max L} W_{L}, \lambda_{\max U} W_{U}\right]
$$

which is equivalent to the following two equations:

$$
\begin{aligned}
& A_{L} W_{L}=\lambda_{\max L} W_{L}, \\
& A_{U} W_{U}=\lambda_{\max U} W_{U}
\end{aligned}
$$

The vector of weight is $\widetilde{\omega}=\left[\alpha W_{L}, \beta W_{U}\right]$, where

$$
\begin{aligned}
& \alpha=\left[\sum_{j=1}^{n} \frac{1}{\sum_{i=1}^{n} a_{i j}^{U}}\right]^{1 / 2}, \\
& \beta=\left[\sum_{j=1}^{n} \frac{1}{\sum_{i=1}^{n} a_{i j}^{L}}\right]^{1 / 2} .
\end{aligned}
$$

2.4. TOPSIS. Assume that a MCDM problem has $n$ alternatives, $A_{1}, \ldots, A_{n}$, and $m$ decision criteria/attributes, $C_{1}$, $\ldots, C_{m}$. Each alternative is evaluated with respect to the $m$ criteria/attributes. All the values/ratings assigned to the alternatives with respect to each criterion form a decision matrix denoted by $X=\left(x_{i j}\right)_{n \times m}$. Let $W=\left(w_{1}, \ldots, w_{m}\right)$ be the relative weight vector about the criteria, satisfying $\sum_{j=1}^{m} w_{j}=1$. Then the TOPSIS method can be summarized as follows.

(1) Normalize the decision matrix $X=\left(x_{i j}\right)_{n \times m}$ using the following equation:

$$
r_{i j}=\frac{x_{i j}}{\sqrt{\sum_{k=1}^{n} x_{k j}^{2}}}, \quad i=1, \ldots, n ; j=1, \ldots, m \text {, }
$$

where $r_{i j}$ is the normalized criteria/attribute value/rating.

(2) Calculate the weighted normalized decision matrix $V=\left(v_{i j}\right)_{n \times m}$ :

$$
v_{i j}=w_{j} r_{i j}, \quad i=1, \ldots, n ; j=1, \ldots, m,
$$

where $w_{j}$ is the relative weight of the $j$ th criterion or attribute, and $\sum_{j=1}^{m} w_{j}=1$.

(3) Determine the ideal and negative-ideal solutions:

$$
\begin{aligned}
A^{*} & =\left\{v_{1}^{*}, \ldots, v_{m}^{*}\right\} \\
& =\left\{\left(\max _{j} v_{i j} \mid j \in \Omega_{b}\right),\left(\min _{j} v_{i j} \mid j \in \Omega_{c}\right)\right\}, \\
A^{-} & =\left\{v_{1}^{-}, \ldots, v_{m}^{-}\right\} \\
& =\left\{\left(\min _{j} v_{i j} \mid j \in \Omega_{b}\right),\left(\max _{j} v_{i j} \mid j \in \Omega_{c}\right)\right\},
\end{aligned}
$$

where $\Omega_{b}$ and $\Omega_{c}$ are the sets of benefit criteria/attributes and cost criteria/attributes, respectively. 
(4) Calculate the Euclidean distances of each alternative from the ideal solution and the negative-ideal solution, respectively:

$$
\begin{gathered}
D_{i}^{*}=\sqrt{\sum_{j=1}^{m}\left(v_{i j}-v_{j}^{*}\right)^{2}}, \quad i=1, \ldots, n, \\
D_{i}^{-}=\sqrt{\sum_{j=1}^{m}\left(v_{i j}-v_{j}^{-}\right)^{2}}, \quad i=1, \ldots, n .
\end{gathered}
$$

(5) Calculate the relative closeness of each alternative to the idea solution. The relative closeness of the alternative $A_{i}$ with respect to $A^{*}$ is defined as

$$
\mathrm{RC}_{i}=\frac{D_{i}^{-}}{D_{i}^{-}+D_{i}^{*}}, \quad i=1, \ldots, n .
$$

(6) Rank the alternatives according to the relative closeness to the ideal solution. The bigger the $\mathrm{RC}_{i}$, the better the alternative $A_{i}$. The best alternative is the one with the greatest relative closeness to the ideal solution.

\section{Mathematic Model of Threat Assessment}

The mathematic model of threat assessment is detailed in this section. As shown in Figure 1, the input of our model includes two parts, namely, subjective factors and objective factors, and the output of this model is the ranking of all the threat objects.

3.1. Integration of Objective Factors. Due to the imprecision of the sensors in the complex situation, sometimes, we should make use of interval numbers to represent the attributive values of the threat targets. In order to measure the different threat degree, the problem arises regarding how best we can aggregate this interval number into a general interval number. The main idea of our proposed method can be shown as follows: this paper integrates the fuzzy numbers through the distance among interval numbers to find a special interval number ( $\left.\left[x_{1}, x_{2}\right], a<x_{1}<x_{2}<b\right)$ whose distance is the optimal value from all other normalized interval numbers with the searching method of genetic algorithms. The binary function $f\left(x_{1}, x_{2}\right)$ is constructed with the summation of all the distance between $\left[x_{1}, x_{2}\right]$ and each normalized interval number. Hence, the problem is mapped into finding the optimal solution of $f\left(x_{1}, x_{2}\right)$ under the constraint $a<x_{1}<$ $x_{2}<b$ with the method of genetic algorithms. This process can be mathematically described by

$$
\begin{aligned}
\min & \mathrm{SD}=\sum_{i=1}^{n} f\left(x_{1}, x_{2}\right)=\sum_{i=1}^{n} D^{2}\left(O, O_{i}\right) \\
\text { s.t. } & O=\left[x_{1}, x_{2}\right] \\
& a \leq x_{1} \leq b \\
& a \leq x_{2} \leq b \\
& x_{1}<x_{2},
\end{aligned}
$$

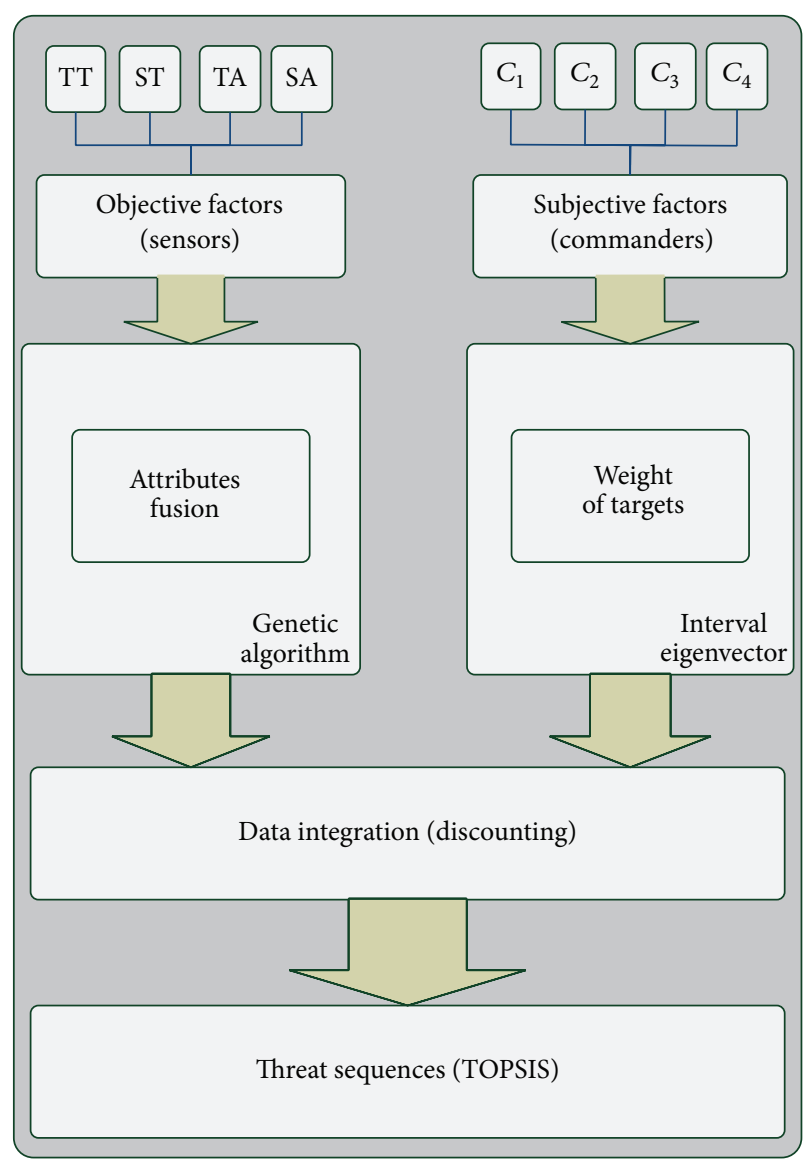

Figure 1: The threat evaluation model.

where $O_{i}$ is the interval value of attribute of the $i$ th object. $D^{2}\left(O, O_{i}\right)$ is the interval distance between $O$ and $O_{i} . a$ and $b$ are the boundary range of $x_{1}$ and $x_{2}$.

Now the procedure of aggregating interval numbers (objective factors) is made by GA in detail as follows.

(1) Determination of the Encoding Method. The first step is to determine the encoding strategy, in other words, how to represent the data between $a$ and $b$ with the computer language. In this paper, the binary encoding strategy is adopted to represent the chromosome. The length of chromosome depends on the accuracy of encoding. Assume that the domain of variable $x_{1}$ is $[a, b]$ and the accuracy of encoding prec is the digit after decimal point. The length of a binary string variable $L$ can be calculated as follows:

$$
L=\max \operatorname{int}\left(\log _{2}^{[(b-a) / \text { prec }+1]}\right),
$$

where prec is to represent the precision of the result and max_int $(x)$ means the maximum integer which is not more than $x$.

(2) Determination of the Decoding Method. The decoding of chromosome is to translate the chromosome from binary data to decimal data in the interval number accordingly. Assume that the binary encoding of a chromosome is represented with $\left(b_{L-1}, \ldots, b_{0}\right)$. Firstly, the binary string $\left(b_{L}, b_{L-1}, \ldots, b_{0}\right)$ should 
be translated to decimal number $x^{\prime}$, which can be denoted as follows:

$$
\left(b_{L}, b_{L-1}, \ldots, b_{0}\right)_{2}=\left(\sum_{i=0}^{L-1} b_{i} \times 2^{i}\right)_{10}=x^{\prime}
$$

Then, the final decoding data in the interval number $[a, b]$ can be calculated as

$$
x=a+x^{\prime} \cdot \frac{b-a}{2^{L}-1}
$$

where $L$ is the encoding length of the chromosome.

(3) Construction of the Initial Population. According to (18), the encoding length of chromosome for each variable can be obtained. Hence, the total length of chromosome for variable weight can be accumulated to be composed of the length of a single long chromosome. For the point position of each chromosome, the method of generating population can be denoted as follows:

$$
b_{i}= \begin{cases}1, & \xi_{i}>0.5 \\ 0, & \xi_{i} \leq 0.5\end{cases}
$$

where $\xi_{i} \in U(0,1)$.

(4) Determination of the Adaptive Function and Adaptive Value. Generally, the adaptive function is designed according to the objective function $f^{*}\left(x_{1}, x_{2}\right)$, and the adaptive function is denoted as $F\left(x_{1}, x_{2}\right)$. In order to lay the foundation for calculating the selected probability of each individual behind, the optimalizing direction of adaptive function $f^{*}\left(x_{1}, x_{2}\right)$ should adapt to the incremental direction of adaptive value. Due to the value of objective function $f^{*}\left(x_{1}, x_{2}\right)>0$, the adaptive function $F\left(x_{1}, x_{2}\right)$ is defined as

$$
F\left(x_{1}, x_{2}\right)=e^{-f^{*}\left(x_{1}, x_{2}\right)}
$$

In this paper, the objective function $f^{*}\left(x_{1}, x_{2}\right)$ is determined by min SD according to (17).

(5) Determination of the Selection Criteria. In this paper, the proportional selection strategy of adaptive value is introduced, and the proportion of every individual is defined as selected probability $P_{i}$. Assume that the population whose scale is $\mathrm{n}$ is given as pop $=\left\{a_{1}, a_{2}, a_{3}, \ldots, a_{n}\right\}$ and the adaptive of $a_{i}$ is given as $F_{i}$; then the selected probability $P_{i}$ is denoted as

$$
P_{i}=\frac{F_{i}}{\sum_{i=1}^{n} F_{i}}, \quad i=1,2,3, \ldots, n .
$$

Then the accumulative probability $Q_{i}$ of every chromosome is denoted as follows:

$$
Q_{i}=\sum_{j=1}^{i} P_{j}, \quad j=1,2, \ldots
$$

After random data $r, r \in[0,1]$ is generated and the selection of chromosome $U_{i}$ for a new population can be made if $Q_{i-1} \leq r \leq Q_{i}$.

(6) Determination of the Genetic Operators. Assume that we have a population $\operatorname{pop}(1)$ including four individuals described as follows:

$$
\begin{aligned}
& \text { pop }(1)=\{\langle 1101011101001100011110\rangle, \\
& \% \% U_{1}\langle 1000011001010001000010\rangle, \\
& \% \% U_{2}\langle 0001100111010110000000\rangle, \\
& \left.\% \% U_{3}\langle 0110101001101110010101\rangle\right\} \% \% U_{4} .
\end{aligned}
$$

After conducting several Roulette Wheel tests, assume that chromosome $U_{2}$ occupies the most area of the whole circle and chromosome $U_{3}$ occupies the least area of the whole circle. According to the selecting criteria, chromosome $U_{2}$ is selected to make a reproduction, while chromosome $U_{3}$ fell into disuse:

$$
\begin{aligned}
& \text { newpop }(1)=\{\langle 1101011101001100011110\rangle, \\
& \% \% U_{1}\langle\mathbf{1 0 0 0 0 1 1 0 0 1 0 1 0 0 0 1 0 0 0 0 1 0}\rangle, \\
& \% \% \mathbf{U}_{2}\langle\mathbf{1 0 0 0 0 1 1 0 0 1 0 1 0 0 0 1 0 0 0 0 1 0}\rangle, \\
& \left.\% \% \mathbf{U}_{3}\langle 0110101001101110010101\rangle\right\} \% \% U_{4} .
\end{aligned}
$$

The crossover operator in this paper adopts the strategy of a single cutting crossover. This method considers the two flanks of the cutting into two substrings; then the right substrings should be exchanged with each other to get two new individuals. If the crossover probability $P_{c}=25 \%$, it means that $25 \%$ of the chromosomes on average exchange each other:

$\langle 110101110$

$1001100011110\rangle$

crossover :

$$
\langle\mathbf{1 0 0 0 0 1 1 0 0} \quad 1010001000010\rangle,
$$

$$
\begin{aligned}
& \text { new }:\langle 1101011101010001000010\rangle, \\
& \text { new }:\langle 1000011001001100011110\rangle .
\end{aligned}
$$

Mutation operator is to change some gene of chromosome with a tiny possibility. If the mutation probability $P_{m}=$ 0.01 , it means that $1 \%$ of all the genes are expected to mutate.

(7) Determine the Process Parameter and Terminal Condition. Through the prior test, the crossover probability $P_{\text {cro }}$ is between 0.4 and 0.99 , the mutation probability $P_{\text {mut }}$ is between 0.0001 and 0.01 , the scale of population is between 20 and 100 , and the terminated condition may be determined by the precise $N$ iterated generation or determined by the minimum bias $\delta$, which satisfies

$$
\mid \text { fitness }_{\max }-\text { fitness }^{*} \mid \leq \delta \text {, }
$$

where fitness ${ }_{\max }$ is the maximum fitness value and fitness ${ }^{*}$ is the objective fitness value. If the judgement condition is not satisfied with the terminal condition, then go to step (4). 
Example 1. Three interval numbers are as follows:

$$
\begin{aligned}
& O_{1}=[5,7], \\
& O_{2}=[5.5,8], \\
& O_{3}=[6,7.5] .
\end{aligned}
$$

Assume that the importance of each interval number is the same. First, we suppose that the integrated interval number of the three fuzzy numbers is represented with $O=$ $\left[x_{1}, x_{2}\right], 4<x_{1}<x_{2}<9$. So the distance between $O$ and each number $O_{i}(i=1,2,3)$ can be shown as follows according to the distance of the interval numbers:

$$
\begin{aligned}
\min f\left(x_{1}, x_{2}\right)= & \sum_{i=1}^{3} D^{2}\left(O, O_{i}\right) \\
= & D^{2}\left(O, O_{1}\right)+D^{2}\left(O, O_{2}\right) \\
& +D^{2}\left(O, O_{3}\right) \\
= & x_{1}^{2}+x_{1} x_{2}+x_{2}^{2}+7 x_{1}-\frac{41}{2} x_{1} \\
& -\frac{37}{2} x_{2}+\frac{769}{6}, \\
& \text { where } 4<x_{1}<x_{2}<9 .
\end{aligned}
$$

Hence, the integration of three interval numbers is mapped into solving the minimum of the formula $f\left(x_{1}, x_{2}\right)$ under the constraint of $4<x_{1}<x_{2}<9$.

Assume that the accuracy of encoding prec is 0.0001 , the crossover probability $p_{\text {cro }}$ is 0.2 , and the mutation probability $p_{\text {mut }}$ is 0.05 . First, the length of chromosome can be calculated as $\log _{2}[(9-4) / 0.0001+1]=16$. Then the encoding length of $x_{1}$ and $x_{2}$ is 32. The adaptive function is $F\left(x_{1}, x_{2}\right)=$ $e^{-f\left(x_{1}, x_{2}\right)}=e^{-\left(x_{1}^{2}+x_{1} x_{2}+x_{2}^{2}+7 x_{1}-(41 / 2) x_{1}-(37 / 2) x_{2}+769 / 6\right)}$. After 300 times of repeat, the convergent result is $(5.4949,7.5023)$ and the minimal value of the objective function is 4.4315 .

3.2. Integration of Subjective Factors. For the subjective factors, the interval AHP (interval eigenvector) is applied to deal with the object's threat weight according to experience of the commanders/experts. A numerical example is used to describe the process as follows.

Example 2. Suppose that we get a pairwise comparison matrix through interval judgements which is denoted as

$$
\widetilde{A}=\left[\begin{array}{ccccc}
{[1,1]} & {[3,4]} & {[2,3]} & {[3,5]} & {[3,5]} \\
{\left[\frac{1}{4}, \frac{1}{3}\right]} & {[1,1]} & {\left[\frac{1}{2}, 1\right]} & {[1,2]} & {[3,5]} \\
{\left[\frac{1}{3}, \frac{1}{2}\right]} & {[1,2]} & {[1,1]} & {[2,3]} & {[2,3]} \\
{\left[\frac{1}{5}, \frac{1}{3}\right]} & {\left[\frac{1}{2}, 1\right]} & {\left[\frac{1}{3}, \frac{1}{2}\right]} & {[1,1]} & {[2,3]} \\
{\left[\frac{1}{5}, \frac{1}{3}\right]} & {\left[\frac{1}{5}, \frac{1}{3}\right]} & {\left[\frac{1}{3}, \frac{1}{2}\right]} & {\left[\frac{1}{3}, \frac{1}{2}\right]} & {[1,1]}
\end{array}\right] .
$$

Then the interval comparison matrix $\widetilde{A}$ can be split into two crisp nonnegative matrices:

$$
\begin{aligned}
A^{L} & =\left[\begin{array}{ccccc}
1 & 3 & 2 & 3 & 3 \\
\frac{1}{4} & 1 & \frac{1}{2} & 1 & 3 \\
\frac{1}{3} & 1 & 1 & 2 & 2 \\
\frac{1}{5} & \frac{1}{2} & \frac{1}{3} & 1 & 2 \\
\frac{1}{5} & \frac{1}{5} & \frac{1}{3} & \frac{1}{3} & 1
\end{array}\right], \\
A^{U} & =\left[\begin{array}{ccccc}
1 & 4 & 3 & 5 & 5 \\
\frac{1}{3} & 1 & 1 & 2 & 5 \\
\frac{1}{2} & 2 & 1 & 3 & 3 \\
\frac{1}{3} & 1 & \frac{1}{2} & 1 & 3 \\
\frac{1}{3} & \frac{1}{3} & \frac{1}{2} & \frac{1}{2} & 1
\end{array}\right] .
\end{aligned}
$$

Then the principal eigenvalue of $\widetilde{A}$ can be calculated: $\lambda_{\max }=\left[\lambda_{\max L}, \lambda_{\max U}\right]$, where $\lambda_{\max L}=4.2892$ and $\lambda_{\max U}=$ 6.3219. The principal right eigenvector corresponding to $\lambda_{\max }$ is $W=\left[W_{L}, W_{U}\right]$, where

$$
\begin{aligned}
& W_{L}=[0.4441,0.1638,0.2072,0.1153,0.0696], \\
& W_{U}=[0.4265,0.1753,0.2113,0.1182,0.0687] .
\end{aligned}
$$

So, the vector of weight $\widetilde{\omega}=\left[\alpha W_{L}, \beta W_{U}\right]$ is

$$
\begin{gathered}
\tilde{\omega}=([0.4052,0.4567],[0.1495,0.1878], \\
{[0.1891,0.2263],[0.1052,0.1266],} \\
[0.0635,0.0735]),
\end{gathered}
$$

where $\alpha=0.9124$ and $\beta=1.0709$.

3.3. Integration of Objective and Subjective Factors. In this part, the method of discounting will be used to integrate the objective and subjective factors. Through the two procedures above, for each threat object, the integrative interval number describing the objective factor can be integrated by the convergent process of genetic algorithm. Hence, assume that there are $n$ threat objects; $n$ optimal interval number will be used to represent the different threat degree; they can be formally denoted as

$$
\begin{aligned}
& \text { Weight (objective) } \\
& \qquad=\left(\left[o_{1}^{-}, o_{1}^{+}\right],\left[o_{2}^{-}, o_{2}^{+}\right], \ldots,\left[o_{n}^{-}, o_{n}^{+}\right]\right) .
\end{aligned}
$$

Sometimes, the commanders need to evaluate the threat degree of each target according to their previous experience in order to control the situation. Hence, through constructing 
the interval decision matrix and calculating the interval eigenvector, the subjective interval numbers representing the evaluation of experts can be described as

$$
\begin{aligned}
& \text { Weight (subjective) } \\
& \qquad=\left(\left[s_{1}^{-}, s_{1}^{+}\right],\left[s_{2}^{-}, s_{2}^{+}\right], \ldots,\left[s_{n}^{-}, s_{n}^{+}\right]\right) .
\end{aligned}
$$

Then the fusion weight of all the threat targets Weight can be calculated according to the method of discounting the objective and subjective factors, which is denoted as

$$
\begin{aligned}
& \text { Weight }=\alpha^{\prime} * \text { Weight (objective) }+\beta^{\prime} \\
& \quad * \text { Weight (subjective) } \\
& \quad=\left(\left[\alpha^{\prime} o_{1}^{-}+\beta^{\prime} s_{1}^{-}, \alpha^{\prime} o_{1}^{+}+\beta^{\prime} s_{1}^{+}\right], \ldots,\right. \\
& \left.\left[\alpha^{\prime} o_{n}^{-}+\beta^{\prime} s_{n}^{-}, \alpha^{\prime} o_{n}^{+}+\beta^{\prime} s_{n}^{+}\right]\right)
\end{aligned}
$$

where $\alpha^{\prime}+\beta^{\prime}=1$. In the process of decision, the discounting factors $\alpha^{\prime}$ and $\beta^{\prime}$ may be modified to adapt the importance of objective factors comparing with subjective factors. If the influence from experts' subjective evaluation is dominant over the influence from the sensors' detection in some complex situation, then the discounting factor should satisfy $\alpha^{\prime}<\beta^{\prime}, \alpha^{\prime}+\beta^{\prime}=1$.

3.4. Ranking the Threat Objects. In this part, the method of TOPSIS will be made use of to deal with the ranking of the threat objects according to their different threat degree. The classical TOPSIS method is a technique for order preference by similarity to ideal solution. The ideal solution (also called positive ideal solution) is a solution that maximizes the benefit criteria/attributes and minimizes the cost criteria/attributes, whereas the negative ideal solution (also called anti-ideal solution) maximizes the cost criteria/attributes and minimizes the benefit criteria/attributes. The so-called benefit criteria/attributes are those for maximization, while the cost criteria/attributes are those for minimization. The best alternative is the one which is closest to the ideal solution and farthest from the negative ideal solution.

Because the normalized interval numbers all distribute in $[0,1]$, the two extreme bounds can be considered as the antiideal solution and the ideal solution. That is to say, the interval number $[0,0]$ can be the anti-ideal solution and the interval number $[1,1]$ can be the ideal solution.

Then the distance between the ideal solution $[1,1]$ and each target's weight from the fusion process of integration with the method of discounting can be calculated. It is not difficult to know that the target nearest to the ideal solution is the most dangerous. Hence the ranking of threat objects can be made easily according to their distance to the ideal solution or the anti-ideal solution.

Example 3. Assume that an interval number $F$ is $[0.4748,0.5152]$ (data from integrating objective and subjective factors); then the distance $D_{F}^{+}$(ideal distance) can be calculated with the interval distance formula $D_{F}^{+}=0.2552$; similarly, the distance $D_{F}^{-}$(anti-ideal distance) also can be
TABLE 1: Interval decision matrix.

\begin{tabular}{lcc}
\hline & $C_{1}$ & $C_{2}$ \\
\hline$A_{1}$ & {$[0.75,1.24]$} & {$[2784,3192]$} \\
$A_{2}$ & {$[1.83,2.11]$} & {$[3671,3857]$} \\
$A_{3}$ & {$[4.90,5.73]$} & {$[4409,4681]$} \\
\hline
\end{tabular}

TABLE 2: Normalized decision matrix.

\begin{tabular}{lcc}
\hline & $C_{1}$ & $C_{2}$ \\
\hline$A_{1}$ & {$[0.55,1.40]$} & {$[0.41,0.50]$} \\
$A_{2}$ & {$[0.33,0.57]$} & {$[0.54,0.60]$} \\
$A_{3}$ & {$[0.12,0.21]$} & {$[0.64,0.73]$} \\
\hline
\end{tabular}

TABLE 3: Aggregated decision matrix.

\begin{tabular}{lc}
\hline & $C$ \\
\hline$A_{1}$ & {$[0.47,0.96]$} \\
$A_{2}$ & {$[0.43,0.59]$} \\
$A_{3}$ & {$[0.31,0.52]$} \\
\hline
\end{tabular}

calculated with interval distance $D_{F}^{-}=0.2452$; at last, the relative closeness $\mathrm{RC}$ can be calculated as

$$
\mathrm{RC}=\frac{D_{F}^{-}}{D_{F}^{-}+D_{F}^{+}}=\frac{0.2452}{0.2452+0.2552}=0.49 .
$$

\section{Numerical Examples}

4.1. The First Numerical Example. In this part, a numerical example [53] is used to illustrate the proposed threat model. Suppose that assessments of the threat objects are all determined with the objective factors $\left(\alpha^{\prime}=1, \beta^{\prime}=0\right)$. The interval decision matrix can be denoted as in Table 1; there are three alternatives $\left(A_{1}, A_{2}, A_{3}\right)$ and two criteria $\left(C_{1}, C_{2}\right)$. The decision maker wants to choose an alternative that has minimum $C_{1}$ and maximum $C_{2}$. Now we order the three alternatives with the proposed model above.

First, the interval decision matrix can be normalized through (1). The normalized decision matrix can be denoted as in Table 2.

Second, through the proposed objective aggregating method with GA, the aggregated results can be converged as in Table 3.

At last, the priority of the three alternatives can be determined by TOPSIS, and the result can be shown as in Table 4 . The ranking of three alternatives is $A_{1}>A_{2}>A_{3}$, which is the same as the result of the paper [53].

This numerical example illustrates the effectiveness of the proposed model without considering the subjective factors. The aggregation of the objective factors is always optimum with GA. In the following part, another numerical example is presented to illustrate the effectiveness of the proposed threat model with considering both objective factors and subjective factors. 
TABLe 4: Priority of decision matrix.

\begin{tabular}{lcccc}
\hline & $C$ & $D^{-}$ & $D^{+}$ & RC \\
\hline$A_{1}$ & {$[0.47,0.96]$} & 0.53 & 0.10 & 0.84 \\
$A_{2}$ & {$[0.43,0.59]$} & 0.26 & 0.24 & 0.52 \\
$A_{3}$ & {$[0.31,0.52]$} & 0.18 & 0.35 & 0.34 \\
\hline
\end{tabular}

TABLE 5: The attributive value of threat objects.

\begin{tabular}{lcccc}
\hline & TT & ST & TA & SA \\
\hline Object 1 (O1) & E & 1500 & {$[160,155]$} & 9 \\
Object 2 (O2) & C & {$[300,450]$} & {$[520,300]$} & {$[11,10]$} \\
Object 3 (O3) & C & {$[420,520]$} & {$[600,300]$} & {$[12,11]$} \\
Object 4 $(O 4)$ & D & 1600 & {$[180,177]$} & 8 \\
Object 5 (O5) & E & {$[600,700]$} & {$[420,300]$} & {$[20,18]$} \\
\hline
\end{tabular}

TABLE 6: Different numerical value of TT.

\begin{tabular}{lcc}
\hline Target style & Threat degree & Details \\
\hline A & 0.8 & Refuelling plane and so forth \\
B & 0.6 & Transport plane and so forth \\
C & 0.9 & Strike aircraft and so forth \\
D & 0.8 & Helicopter \\
E & 1.0 & Missile and so forth \\
F & 0.2 & Fictional goal and so forth \\
\hline
\end{tabular}

4.2. The Second Numerical Example. In this part, a real application in aerial object threat will be introduced to describe the operational procedure of threat assessment. The threat objects and their attributes can be shown as in Table 5.

In Table 5, TT, ST, TA, and SA mean "type of target," "speed of target," "time of arrival," and "shortcut of airway." At the same time TT, ST are benefit attributes, TA, SA are cost attributes, and the numerical value TT is described in Table 6.

In order to eliminate the influence resulting from different measures, the normalization can be made through (1). The normalized result of Table 5 can be described as in Table 7 .

Through the objective fusion method by GA, the integrated results can be converged as in Table 8 .

In the following part, we will take the commanders' experience into consideration. The interval decision by experts for the five threat objects is described as

$$
\left[\begin{array}{lllll}
{[1,1]} & {[3,4]} & {[2,3]} & {[3,5]} & {[3,5]} \\
{\left[\frac{1}{4}, \frac{1}{3}\right]} & {[1,1]} & {\left[\frac{1}{2}, 1\right]} & {[1,2]} & {[3,5]} \\
{\left[\frac{1}{3}, \frac{1}{2}\right]} & {[1,2]} & {[1,1]} & {[2,3]} & {[2,3]} \\
{\left[\frac{1}{5}, \frac{1}{3}\right]} & {\left[\frac{1}{2}, 1\right]} & {\left[\frac{1}{3}, \frac{1}{2}\right]} & {[1,1]} & {[2,3]} \\
{\left[\frac{1}{5}, \frac{1}{3}\right]} & {\left[\frac{1}{5}, \frac{1}{3}\right]} & {\left[\frac{1}{3}, \frac{1}{2}\right]} & {\left[\frac{1}{3}, \frac{1}{2}\right]} & {[1,1]}
\end{array}\right]
$$

As for the subjective factors, the method of interval eigenvector is used to generate the interval threat weights of all targets. The fusion process of subjective factors is like
TABLE 7: Normalized attribute value of threat objects.

\begin{tabular}{ccccc}
\hline & TT & ST & TA & SA \\
\hline O1 & {$[0.48,0.48]$} & {$[0.62,0.64]$} & {$[0.71,0.60]$} & {$[0.52,0.50]$} \\
O2 & {$[0.44,0.44]$} & {$[0.16,0.19]$} & {$[0.37,0.18]$} & {$[0.47,0.41]$} \\
O3 & {$[0.44,0.44]$} & {$[0.17,0.22]$} & {$[0.37,0.16]$} & {$[0.43,0.38]$} \\
O4 & {$[0.48,0.48]$} & {$[0.67,0.68]$} & {$[0.62,0.54]$} & {$[0.58,0.56]$} \\
O5 & {$[0.39,0.39]$} & {$[0.25,0.30]$} & {$[0.37,0.23]$} & {$[0.26,0.23]$} \\
\hline
\end{tabular}

TABLE 8: Integrated result of attributes of each corresponding object.

\begin{tabular}{lcc}
\hline & Fusion data & $\operatorname{minSD}$ \\
\hline O1 & {$[0.58,0.60]$} & 0.0114 \\
O2 & {$[0.28,0.31]$} & 0.0364 \\
O3 & {$[0.25,0.38]$} & 0.0311 \\
O4 & {$[0.57,0.60]$} & 0.0101 \\
O5 & {$[0.30,0.31]$} & 0.0318 \\
\hline
\end{tabular}

TABle 9: Fusion between objective and subjective weight $(\mathrm{O}(w)$ : objective weight, $\mathrm{S}(w)$ : subjective weight, and $\mathrm{I}(w)$ : integrated weight), $\alpha=0.6$.

\begin{tabular}{lccc}
\hline & $\mathrm{O}(w)$ & $\mathrm{S}(w)$ & $\mathrm{I}(w)$ \\
\hline O1 & {$[0.58,0.60]$} & {$[0.41,0.46]$} & {$[0.51,0.54]$} \\
O2 & {$[0.28,0.31]$} & {$[0.15,0.19]$} & {$[0.23,0.26]$} \\
O3 & {$[0.25,0.38]$} & {$[0.19,0.23]$} & {$[0.23,0.32]$} \\
O4 & {$[0.57,0.60]$} & {$[0.11,0.13]$} & {$[0.39,0.41]$} \\
O5 & {$[0.30,0.31]$} & {$[0.06,0.07]$} & {$[0.20,0.21]$} \\
\hline
\end{tabular}

TABLE 10: Relative closeness (RC) of all targets: $D_{F}^{+}$means the ideal distance, $D_{F}^{-}$means the anti-ideal distance, and $\mathrm{RC}$ means relative closeness.

\begin{tabular}{lcccc}
\hline & $\mathrm{I}(w)$ & $D_{F}^{+}$ & $D_{F}^{-}$ & $\mathrm{RC}$ \\
\hline O1 & {$[0.51,0.54]$} & 0.3194 & 0.1894 & 0.3722 \\
O2 & {$[0.23,0.26]$} & 0.5701 & 0.0601 & 0.0954 \\
O3 & {$[0.23,0.32]$} & 0.5293 & 0.0753 & 0.1245 \\
O4 & {$[0.39,0.41]$} & 0.3613 & 0.1593 & 0.3060 \\
O5 & {$[0.20,0.21]$} & 0.6257 & 0.0437 & 0.0653 \\
\hline
\end{tabular}

Example 2, and the fusion weight of threat objects by experts' decision is

$$
\begin{gathered}
\widetilde{\omega}=([0.41,0.46],[0.15,0.19],[0.19,0.23], \\
[0.11,0.13],[0.06,0.07]) .
\end{gathered}
$$

Hence, at present, the integration between objective factors and subjective factors can be made by the method of discounting. It can be denoted as in Table 9.

According to the idea of TOPSIS, we can get the ideal distance $D_{F}^{+}$and anti-ideal distance $D_{F}^{-}$of each target's fusion weight, and the ideal interval number is $[1,1]$ and the antiideal interval number is $[0,0]$, so we can get the relative closeness of each target described as in Table 10. According to the relative closeness $\mathrm{RC}$, the ranking of all threat targets is $\mathrm{O}_{1}>\mathrm{O}_{4}>\mathrm{O}_{3}>\mathrm{O}_{2}>\mathrm{O}_{5}$ (threat degree from large to small). This result is shown by Figure 2 explicitly. 

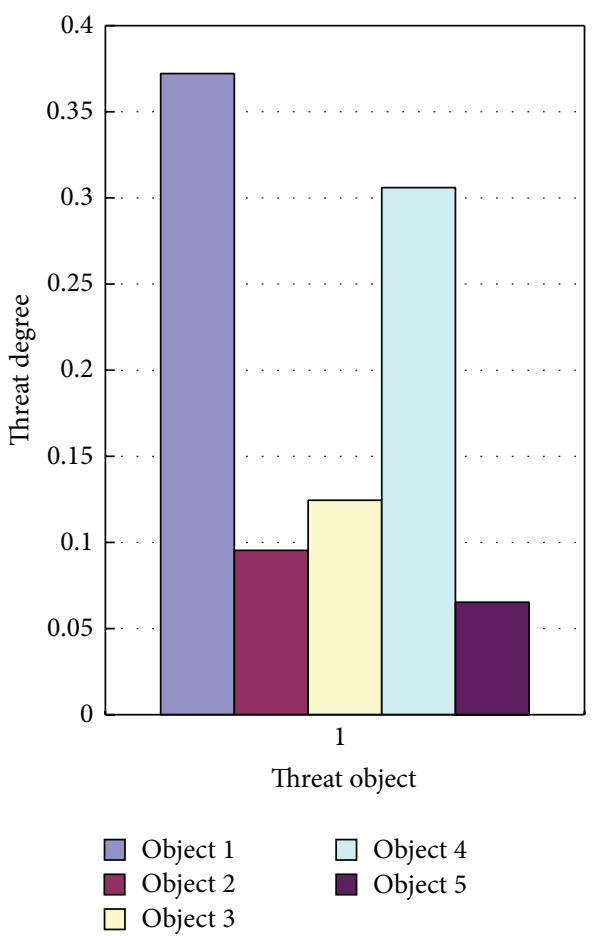

FIgURE 2: The threat degree of all threat objects.

As presented above, the proposed threat model can not only be applied to the objective issue, but can also be easily extended to considering the subjective issue from knowledge of the domain experts. When the objective discounting factor $\alpha^{\prime}=1$ and the subjective factor $\beta^{\prime}=0$, the proposed threat model can be absolutely applied to the objective issues. When the objective discounting factor $\alpha^{\prime}=0$ and the subjective factor $\beta^{\prime}=1$, the proposed threat model can be absolutely applied to the subjective issues. Otherwise, the proposed threat model is applied in both subjective and objective issues according to the optimum discounting factors $\left(\alpha^{\prime}\right.$ and $\left.\beta^{\prime}\right)$.

\section{Conclusion}

Threat assessment is very important in many fields. This paper proposes a new model of threat assessment based on interval number. Both subjective and objective factors are taken into consideration in this threat assessment model. GA is used to determine the objective factors. In addition, interval AHP is applied to determine subjective factors. Then a method of discounting is proposed to integrate the subjective weight and objective weight. At last, the idea of TOPSIS is adopted to rank the objects according to their threat degree. A numerical example and a real application are used to illustrate the effectiveness of the proposed threat model.

\section{Conflict of Interests}

The author declares that there is no conflict of interests regarding the publication of this paper.

\section{Acknowledgments}

The author greatly appreciates the reviewers' valuable suggestions. This work is partially supported by National High Technology Research and Development Program of China (863 Program) (Grant no. 2013AA013801), National Natural Science Foundation of China (Grant no. 61174022), Specialized Research Fund for the Doctoral Program of Higher Education (Grant no. 20131102130002), R\&D Program of China (2012BAH07B01), and the Open Funding Project of State Key Laboratory of Virtual Reality Technology and Systems, Beihang University (Grant no. BUAA-VR-14KF-02).

\section{References}

[1] T. L. Saaty, "How to make a decision: the analytic hierarchy process," European Journal of Operational Research, vol. 48, pp. 9-26, 1990.

[2] T. L. Saaty, "The modern science of multicriteria decision making and its practical applications: the AHP/ANP approach," Operations Research, vol. 61, no. 5, pp. 1101-1118, 2013.

[3] D. J. Hall and R. A. Davis, "Engaging multiple perspectives: a value-based decision-making model," Decision Support Systems, vol. 43, no. 4, pp. 1588-1604, 2007.

[4] Y. Zhang, Z.-P. Fan, and Y. Liu, "A method based on stochastic dominance degrees for stochastic multiple criteria decision making," Computers \& Industrial Engineering, vol. 58, no. 4, pp. 544-552, 2010.

[5] N. Chiadamrong, "An integrated fuzzy multi-criteria decision making method for manufacturing strategies selection," Computers \& Industrial Engineering, vol. 37, no. 1, pp. 433-436, 1999.

[6] C.-C. Hon, Y.-Y. Guh, K.-M. Wang, and E. S. Lee, "Fuzzy multiple attributes and multiple hierarchical decision making," Computers \& Mathematics with Applications, vol. 32, no. 12, pp. 109119, 1996.

[7] G. A. Forgionne and R. Kohli, "A multiple criteria assessment of decision technology system journal quality," Information \& Management, vol. 38, no. 7, pp. 421-435, 2001.

[8] Z.-P. Fan, Y. Liu, and B. Feng, "A method for stochastic multiple criteria decision making based on pairwise comparisons of alternatives with random evaluations," European Journal of Operational Research, vol. 207, no. 2, pp. 906-915, 2010.

[9] B. Al-Najjar and I. Alsyouf, "Selecting the most efficient maintenance approach using fuzzy multiple criteria decision making," International Journal of Production Economics, vol. 84, no. 1, pp. 85-100, 2003.

[10] J. Q. Wang, "Multi-criteria decision-making approach with incomplete certain information based on ternary AHP," Journal of Systems Engineering and Electronics, vol. 17, no. 1, pp. 109-114, 2006.

[11] F. T. S. Chan, N. Kumar, M. K. Tiwari, H. C. W. Lau, and K. L. Choy, "Global supplier selection: a fuzzy-AHP approach," International Journal of Production Research, vol. 46, no. 14, pp. 38253857, 2008

[12] C. Kahraman, S. C. Onar, and B. Oztaysi, "Fuzzy multicriteria decisionmaking: a literature review," International Journal of Computational Intelligence Systems, vol. 8, no. 4, pp. 637-666, 2015.

[13] H.-C. Liu, L. Liu, and Q.-L. Lin, "Fuzzy failure mode and effects analysis using fuzzy evidential reasoning and belief rule-based 
methodology," IEEE Transactions on Reliability, vol. 62, no. 1, pp. 23-36, 2013.

[14] Y. Wang, Y. Dai, Y.-W. Chen, and F. Meng, “The evidential reasoning approach to medical diagnosis using intuitionistic fuzzy Dempster-Shafer theory," International Journal of Computational Intelligence Systems, vol. 8, no. 1, pp. 75-94, 2015.

[15] H.-C. Liu, J.-X. You, X.-Y. You, and M.-M. Shan, "A novel approach for failure mode and effects analysis using combination weighting and fuzzy VIKOR method," Applied Soft Computing Journal, vol. 28, pp. 579-588, 2015.

[16] Y. Deng, R. Sadiq, W. Jiang, and S. Tesfamariam, "Risk analysis in a linguistic environment: a fuzzy evidential reasoning-based approach," Expert Systems with Applications, vol. 38, no. 12, pp. 15438-15446, 2011.

[17] W. Jiang, Y. Yang, Y. Luo, and X. Qin, "Determining basic probability assignment based on the improved similarity measures of generalized fuzzy numbers," International Journal of Computers Communications \& Control, vol. 10, no. 3, pp. 333-347, 2015.

[18] W. Jiang, Y. Luo, X. Qin, and J. Zhan, "An improved method to rank generalized fuzzy numbers with different left heights and right heights," Journal of Intelligent \& Fuzzy Systems, vol. 28, no. 5, pp. 2343-2355, 2015.

[19] C. Fu and K.-S. Chin, "Robust evidential reasoning approach with unknown attribute weights," Knowledge-Based Systems, vol. 59, pp. 9-20, 2014.

[20] H.-C. Liu, J.-X. You, X.-J. Fan, and Q.-L. Lin, "Failure mode and effects analysis using D numbers and grey relational projection method," Expert Systems with Applications, vol. 41, no. 10, pp. 4670-4679, 2014.

[21] Y. Deng, "Generalized evidence theory," Applied Intelligence, 2015.

[22] Y. Deng, S. Mahadevan, and D. Zhou, "Vulnerability assessment of physical protection systems: a bio-inspired approach," International Journal of Unconventional Computing, vol. 11, 2015.

[23] B. Kang, Y. Deng, R. Sadiq, and S. Mahadevan, "Evidential cognitive maps," Knowledge-Based Systems, vol. 35, no. 15, pp. 7786, 2012.

[24] X. Su, S. Mahadevan, P. Xu, and Y. Deng, "Dependence assessment in human reliability analysis using evidence theory and AHP," Risk Analysis, vol. 35, no. 7, pp. 1296-1316, 2015.

[25] X. Deng, Y. Hu, Y. Deng, and S. Mahadevan, "Supplier selection using AHP methodology extended by D numbers," Expert Systems with Applications, vol. 41, no. 1, pp. 156-167, 2014.

[26] W. Pedrycz, S. C. Chen, S. H. Rubin, and G. Lee, "Risk evaluation through decision-support architectures in threat assessment and countering terrorism," Applied Soft Computing Journal, vol. 11, no. 1, pp. 621-631, 2011.

[27] B. van de Walle and A.-F. Rutkowski, "A fuzzy decision support system for IT service continuity threat assessment," Decision Support Systems, vol. 42, no. 3, pp. 1931-1943, 2006.

[28] C. G. Looney and L. R. Liang, "Cognitive situation and threat assessments of ground battlespaces," Information Fusion, vol. 4, no. 4, pp. 297-308, 2003.

[29] I. M. Khadam and J. J. Kaluarachchi, "Multi-criteria decision analysis with probabilistic risk assessment for the management of contaminated ground water," Environmental Impact Assessment Review, vol. 23, no. 6, pp. 683-721, 2003.

[30] T. Assmuth and M. Hildén, "The significance of information frameworks in integrated risk assessment and management," Environmental Science \& Policy, vol. 11, no. 1, pp. 71-86, 2008.
[31] Y.-C. Chen and H.-W. Ma, "Combining the cost of reducing uncertainty with the selection of risk assessment models for remediation decision of site contamination," Journal of Hazardous Materials, vol. 141, no. 1, pp. 17-26, 2007.

[32] K.-S. Jeong, K.-W. Lee, and H.-K. Lim, "Risk assessment on hazards for decommissioning safety of a nuclear facility," Annals of Nuclear Energy, vol. 37, no. 12, pp. 1751-1762, 2010.

[33] E. J. Bonano, G. E. Apostolakis, P. F. Salter, A. Ghassemi, and S. Jennings, "Application of risk assessment and decision analysis to the evaluation, ranking and selection of environmental remediation alternatives," Journal of Hazardous Materials, vol. 71, no. 1-3, pp. 35-57, 2000.

[34] F. H. van Duijne, D. van Aken, and E. G. Schouten, "Considerations in developing complete and quantified methods for risk assessment," Safety Science, vol. 46, no. 2, pp. 245-254, 2008.

[35] M. Kalantarnia, F. Khan, and K. Hawboldt, "Dynamic risk assessment using failure assessment and Bayesian theory," Journal of Loss Prevention in the Process Industries, vol. 22, no. 5, pp. 600-606, 2009.

[36] Y. Deng, W. Jiang, and R. Sadiq, "Modeling contaminant intrusion in water distribution networks: a new similarity-based DST method," Expert Systems with Applications, vol. 38, no. 1, pp. 571578, 2011.

[37] S. B. Tsai, M. F. Chien, Y. Xue et al., "Using the fuzzy DEMATEL to determine environmental performance: a case of printed circuit board industry in Taiwan," PLOS ONE, vol. 10, no. 6, Article ID e0129153, 2015.

[38] D. Liang, D. Liu, W. Pedrycz, and P. Hu, "Triangular fuzzy decision-theoretic rough sets," International Journal of Approximate Reasoning, vol. 54, no. 8, pp. 1087-1106, 2013.

[39] S. B. Tsai, Y. Z. Xue, P. Y. Huang et al., "Establishing a criteria system for green production," Proceedings of the Institution of Mechanical Engineers, Part B: Journal of Engineering Manufacture, 2014.

[40] K. Kimiafar, F. Sadoughi, A. Sheikhtaheri, and M. Sarbaz, "Prioritizing factors influencing nurses' satisfaction with hospital information systems: a fuzzy analytic hierarchy process approach," Computers Informatics Nursing, vol. 32, no. 4, pp. 174-181, 2014.

[41] Z. I. Petrou, V. Kosmidou, I. Manakos et al., "A rule-based classification methodology to handle uncertainty in habitat mapping employing evidential reasoning and fuzzy logic," Pattern Recognition Letters, vol. 48, pp. 24-33, 2014.

[42] R. E. Moore, Interval arithmetic and automatic error analysis in digital computing [Ph.D. thesis], Stanford University, Stanford, Calif, USA, 1962.

[43] R. E. Moore, Interval Analysis, Prentice Hall, Englewood Cliffs, NJ, USA, 1966.

[44] C. Jiang, X. Han, F. J. Guan, and Y. H. Li, "An uncertain structural optimization method based on nonlinear interval number programming and interval analysis method," Engineering Structures, vol. 29, no. 11, pp. 3168-3177, 2007.

[45] A. Sengupta and T. K. Pal, "On comparing interval numbers," European Journal of Operational Research, vol. 127, no. 1, pp. 2843, 2000.

[46] Z. Yue, "An extended TOPSIS for determining weights of decision makers with interval numbers," Knowledge-Based Systems, vol. 24, no. 1, pp. 146-153, 2011.

[47] L. Tran and L. Duckstein, "Comparison of fuzzy numbers using a fuzzy distance measure," Fuzzy Sets and Systems, vol. 130, no. 3, pp. 331-341, 2002. 
[48] A. Brdossy, R. Hagaman, L. Duckstein, and I. Bogardi, Fuzzy Least Squares Regression: Theory and Application, Omnitech Press, Warsaw, Poland; Physica, Heidelberg, Germany, 1992.

[49] P. Diamond, "Fuzzy least squares," Information Sciences, vol. 46, no. 3, pp. 141-157, 1988.

[50] P. Diamond and R. Körner, "Extended fuzzy linear models and least squares estimates," Computers and Mathematics with Applications, vol. 33, no. 9, pp. 15-32, 1997.

[51] C. Bertoluzza, N. C. Blanco, and A. Salas, "On a new class of distances between fuzzy numbers," Mathware \& Soft Computing, vol. 2, no. 2, pp. 71-84, 1995.

[52] F. T. S. Chan, S. H. Chung, and P. L. Y. Chan, "An adaptive genetic algorithm with dominated genes for distributed scheduling problems," Expert Systems with Applications, vol. 29, no. 2, pp. 364-371, 2005.

[53] M. K. Sayadi, M. Heydari, and K. Shahanaghi, "Extension of vikor method for decision making problem with interval numbers," Applied Mathematical Modelling, vol. 33, no. 5, pp. 22572262, 2009. 


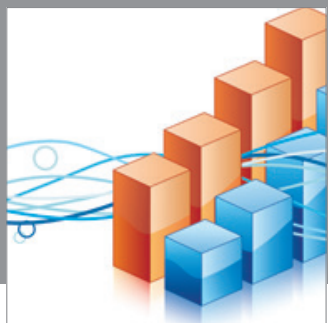

Advances in

Operations Research

mansans

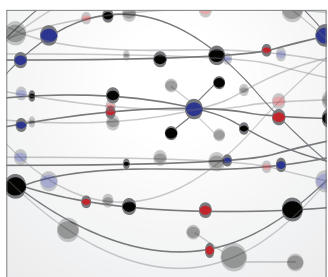

The Scientific World Journal
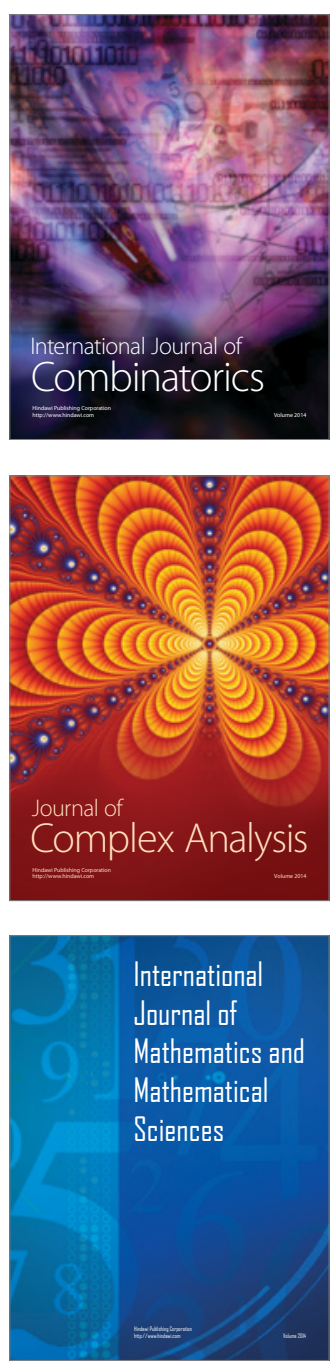
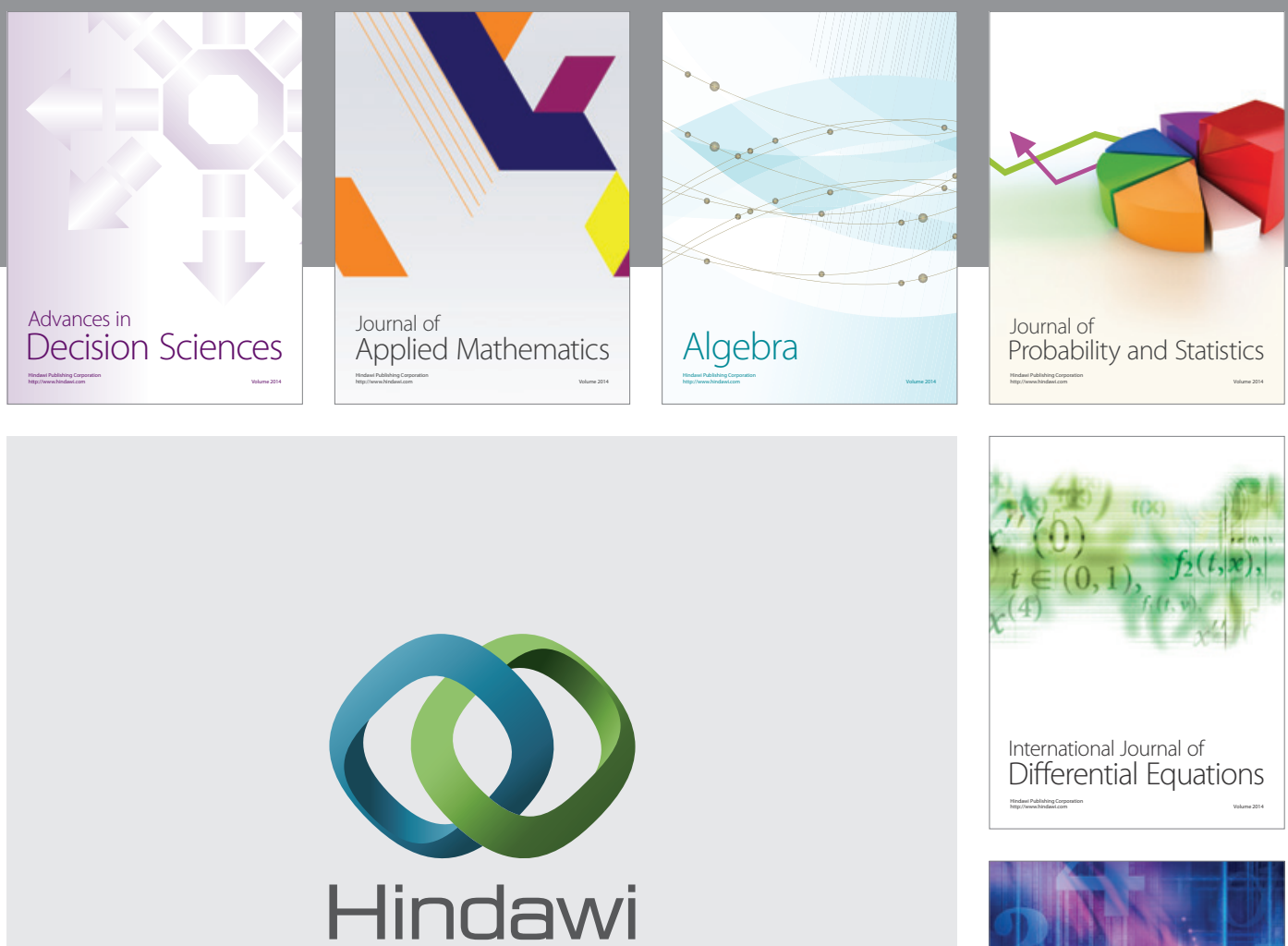

Submit your manuscripts at http://www.hindawi.com
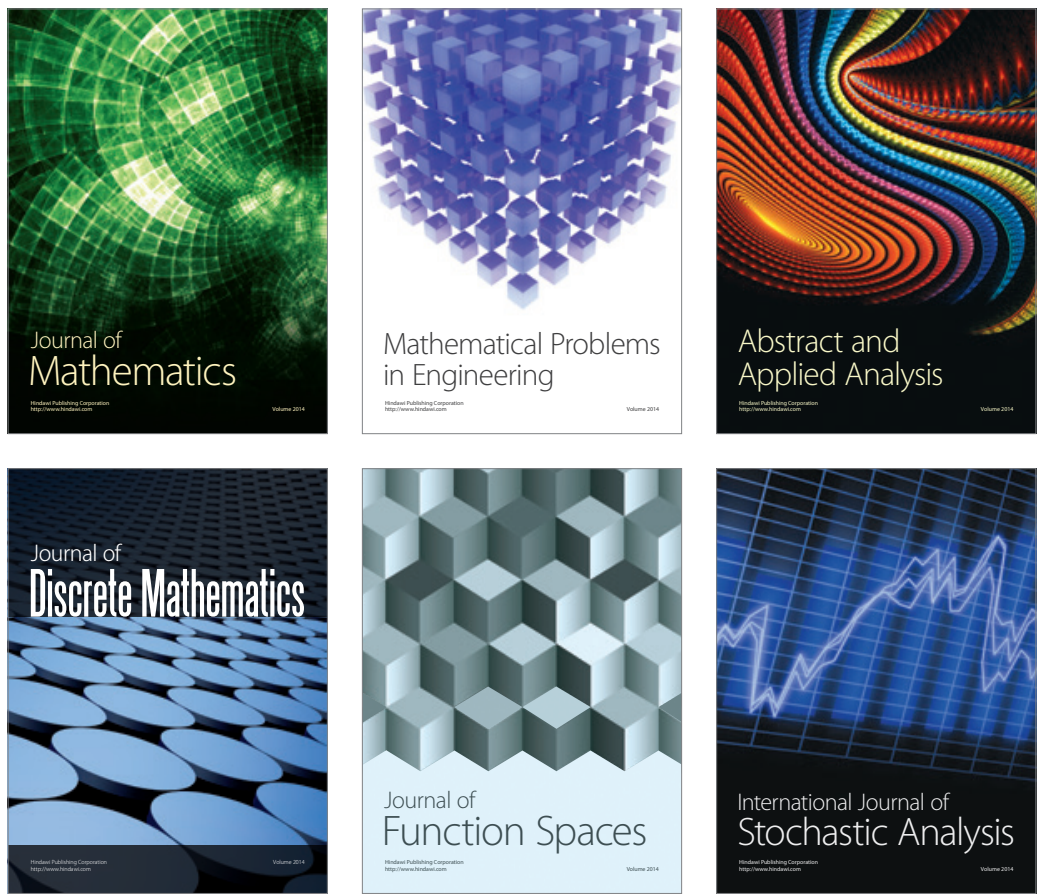

Journal of

Function Spaces

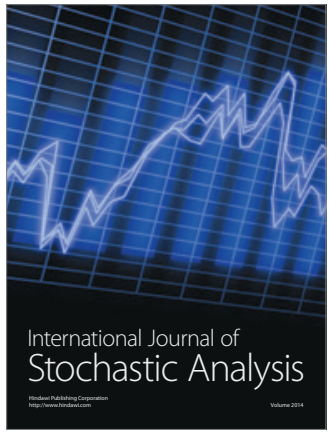

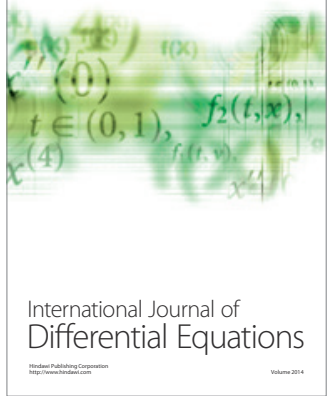
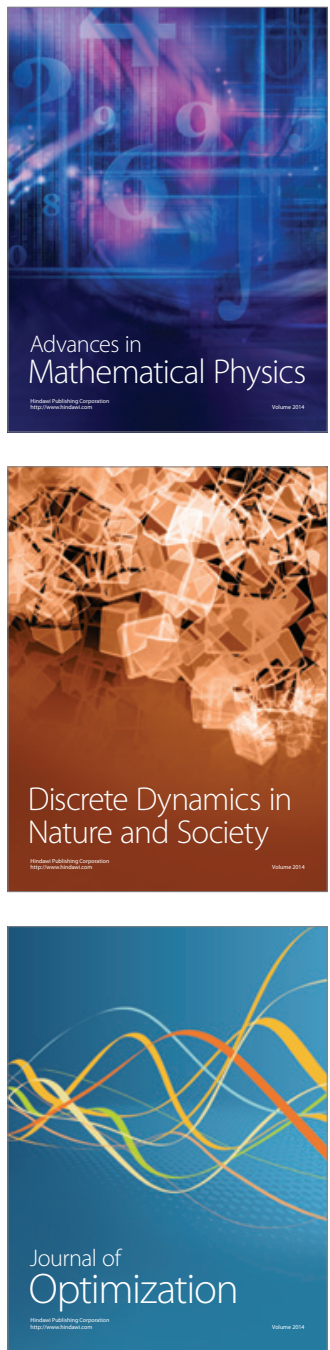